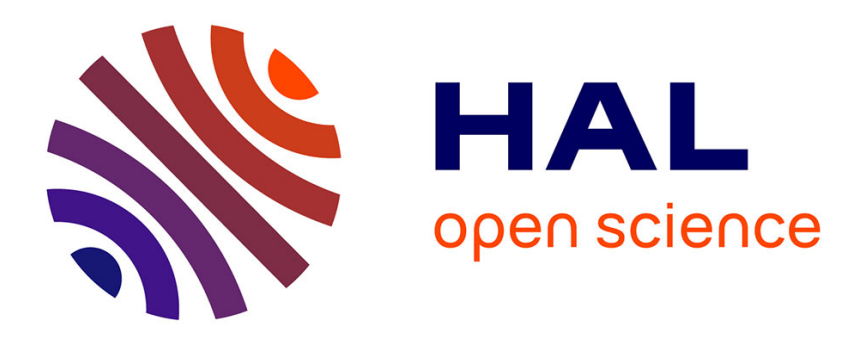

\title{
A generalization of the Leman-Weaire-Thorpe theorem
}

\author{
O. Betbeder-Matibet, M. Hulin
}

\section{To cite this version:}

O. Betbeder-Matibet, M. Hulin. A generalization of the Leman-Weaire-Thorpe theorem. Journal de Physique, 1979, 40 (9), pp.901-912. 10.1051/jphys:01979004009090100 . jpa-00209175

\section{HAL Id: jpa-00209175 https://hal.science/jpa-00209175}

Submitted on 1 Jan 1979

HAL is a multi-disciplinary open access archive for the deposit and dissemination of scientific research documents, whether they are published or not. The documents may come from teaching and research institutions in France or abroad, or from public or private research centers.
L'archive ouverte pluridisciplinaire HAL, est destinée au dépôt et à la diffusion de documents scientifiques de niveau recherche, publiés ou non, émanant des établissements d'enseignement et de recherche français ou étrangers, des laboratoires publics ou privés. 


\title{
A generalization of the Leman-Weaire-Thorpe theorem
}

\author{
O. Betbeder-Matibet and M. Hulin \\ Groupe de Physique des Solides de l'Ecole Normale Supérieure (*), \\ Université Paris VII, 2, place Jussieu, 75221 Paris Cedex 05, France \\ (Reçu le 2 mars 1979, révisé le 7 mai 1979, accepté le 15 mai 1979)
}

\begin{abstract}
Résumé. - Nous avons généralisé un résultat, initialement démontré par Leman, Weaire et Thorpe pour des semiconducteurs tétracoordonnés, à un ensemble de situations très diverses où le désordre topologique est le principal responsable des propriétés électroniques du système. Nous considérons des matériaux constitués d'amas identiques d'atomes reliés par des chaînes d'atomes elles-mêmes identiques et tels que les interactions entre ces entités soient uniquement fonction de leur position relative sur le graphe associé à l'ensemble de la structure. Nous montrons qu'il est alors possible de factoriser le Hamiltonien à liaisons fortes ; on obtient ainsi une densité d'états en énergie constituée de " pics delta " et de bandes d'énergie ; celles-ci peuvent être déterminées, par une transformation algébrique simple, à partir du spectre de la matrice incidence associée au graphe qui admet les amas pour sommets et les chaînes atomiques comme arêtes. Cette propriété est étendue au cas où les amas sont de deux types, les liaisons n'intervenant qu'entre amas de types différents. Pour donner un exemple d'application, nous avons repris les calculs de structure de bande de $\mathrm{GeSe}_{2}$ publiés par Lannoo et Bensoussan, et montré qu'on peut améliorer leur traitement en tenant compte par exemple du couplage entre orbitales liantes et antiliantes adjacentes à un même atome de germanium.
\end{abstract}

\begin{abstract}
A result of Leman, Weaire and Thorpe, initially proved for fourfold coordinated semiconductors is generalized to much wider situations where topological disorder may be taken as the primary factor determining the electronic properties of the system. We consider materials where identical clusters of atoms are linked through identical atomic chains, and where the interactions between these entities only depend on their relative positions on the graph underlying the whole structure. We show that there is a possible factorization of the tight-binding Hamiltonian which yields an energy density of states made up of delta peaks and energy bands; these bands, in turn, can be obtained, through a simple algebraic manipulation, from the spectrum of the adjacency matrix of the graph which represents the clusters as vertices and the chains as edges. This property is further generalized to the case where clusters are of two types, with chains only between clusters of different types. In order to provide an illustrative application of our formalism, we repeat band structure calculations for $\mathrm{GeSe}_{2}$ published by Lannoo and Bensoussan and show that more precise results can now easily be obtained, for instance, by taking into account the interaction between bonding and antibonding orbitals adjacent to the same $\mathrm{Ge}$ atom.
\end{abstract}

1. Introduction. - Several years ago, Weaire and Thorpe $[1,2]$ rediscovered and generalized a result which had been previously obtained by Leman [3] in a restricted context : they showed that the electronic energy spectrum, in a four fold-coordinated covalent semiconductor described by a tight-binding Hamiltonian, with four equivalent $\mathrm{sp}^{3}$ orbitals belonging to the same atom, consists of isolated energy levels ( $\delta$-peak » contributions to the energy density of states) plus energy bands; the energies of the latter can be readily obtained from the spectrum of

(*) Laboratoire associé au Centre National de la Recherche Scientifique. the adjacency matrix of the graph having the atoms as vertices and the covalent bonds between nearest neighbours as edges [4]. This " Leman-WeaireThorpe" (L.W.T.) theorem applies equally well to topologically disordered materials and to crystals, and, for the former, can be used to show that forbidden energy bands remain when the crystal periodicity is lost, provided the ensuing disorder is purely topological (which means that no dangling bond appears and that all atoms and bonds remain equivalent to one another).

The initial demonstration by Weaire and Thorpe was somewhat clumsy, and was given anew in a much simplified form by these authors [5], as well as by some others $[6,7]$. Various attempts to gene- 
ralize the L.W.T. theorem have also been put forward $[8,9]$. Some of them make it possible to use this result in situations which are more complicated from a structural and chemical point of view. An example is provided by the Ge-Se system [10] : 4-fold coordinated germanium atoms may there be connected by selenium chains of various lengths, depending on the selenium concentration ; for some critical concentrations, all chains have essentially the same length and one comes very close to a situation of pure topological disorder.

The aim of this paper is to provide as complete a generalization as possible of the L.W.T. result. Keeping within the framework of a tight-binding one-electron Hamiltonian, we shall first treat the problem of a structure which can be described starting from some regular graph (in which every vertex participates in the same number, say $Z$, of edges), but where the vertices and the edges now correspond, in the material, to composite entities including clusters or chains of several atoms ; moreover, some of the atomic orbitals associated with these atoms may correspond to free orbitals (for instance lone pairs), not involved in covalent bonds. A second situation will also be examined, for which the graph is bipartite : vertices are of two kinds, the corresponding atomic clusters having possibly different structures and properties, and bonding in the graph only appears between vertices of different kinds.

These refinements offer the possibility of using the L.W.T. theorem in a much widened range of physical systems.

Rather stringent conditions on bond lengths and angles reduce the possibility of appearance of topological disorder for a covalent semi-conductor with only one species of, say, 4-fold coordinated atoms. These conditions are much relaxed when bonding between vertices may be realized through chains of atoms which can be rotated and twisted in various ways.

Thus, admitting more complex actual structures for the atomic clusters or chains which are respectively associated with the vertices and edges of a graph, considerably enlarges the number of situations where topological disorder may be considered as the primary factor determining the electronic properties of a random system.

2. Systems with one type of vertices. - 2.1 DESCRIPTION OF THE SYSTEMS. - A system of the kind we study here will be associated with a regular graph $\mathrm{G}$, and described by a tight-binding Hamiltonian $H$, the matrix elements of which are entirely determined once we know :

- the structure of the atomic clusters and chains we respectively associate with the vertices and edges of $\mathrm{G}$,
- the interactions between atomic orbitals belonging to these clusters and chains.

We aim at relating $H$ to the various matrices, including the adjacency matrix $C$, describing the graph G. This is possible only if :

(i) all clusters and all chains are identical; as a consequence any chain between two vertices must be symmetrical with respect to its middle point : ortherwise a dissymmetry would be introduced between the vertices at both extremities of the chain;

(ii) all interactions between orbitals belonging to these entities only depend on the positions of the orbitals within the cluster or chain which they belong to, and on the relative positions on the graph $G$, of these clusters and/or chains.

Atomic orbitals will be distributed into two classes :

- «vertex orbitals" (V.O.) : this class only contains orbitals that belong to atoms in vertex clusters and play a totally isotropic role within the structure (for instance, lone pair orbitals, interacting identically with all other orbitals involved in covalent bonds); we shall introduce $n$ orbitals of this type for every vertex of $\mathrm{G}$;

- « edge-orbitals" (E.O.) : this class, which gathers all other atomic orbitals, includes those associated with atoms within the edge chains and those belonging to atoms in the vertex clusters which have a preferential connection with one edge (for instance $\mathrm{sp}^{3}$ orbitals in a 4-fold coordinated semiconductor will here be considered as «edge orbitals $»)$.

Actually, the symmetry of edge chains, which we stressed above, suggests the definition of symmetrical and antisymmetrical combinations of the atomic E.O.'s (the symmetry still being understood with respect to the middle point of the chain). To every edge will thus correspond $m_{\mathrm{S}}$ symmetrical E.O.'s (S.E.O.) and $m_{\mathrm{A}}$ antisymmetrical E.O.'s (A.E.O.). We shall take $m=m_{\mathrm{S}}+m_{\mathrm{A}}$. The S.E.O.'s generalize the conventional bonding orbitals associated with covalent bonds; but they may also include lone pair orbitals possibly belonging to some atom at the middle point of the chain. The A.E.O.'s, on the other hand, are reminiscent of antibonding orbitals in covalent bonding. Some coherence must be introduced in their definition : all edges of the graph should be oriented, in a completely arbitrary way. For a given edge, A.E.O.'s will be systematically defined for any pair of atomic orbitals symmetrical with respect to the edge middle point, by taking the orbital on the side of the starting point vertex minus the orbital on the side of the end point vertex, and then normalizing.

All the preceding conditions and conventions provide us with the necessary material to treat our problem. We shall only consider cases where interaction is restricted to orbitals belonging to adjacent components of the underlying graph $\mathrm{G}$ : vertices at 
the extremities of some common edge, or edges sharing a common vertex. This will simplify the exposition but does not correspond to an actual limitation of the generality of our results. We shall later indicate possible generalizations.

2.2 Hamiltonian StruCtURE. - Using adequate periodic boundary conditions, we shall restrict consideration to a set of $N$ vertices, linked by $M$ edges, with :

$$
M=\frac{Z N}{2}>N
$$

The hamiltonian $H$ will be decomposed into four submatrices, after the orbital basis for the tightbinding treatment of the system has been ordered in such a way that V.O.'s appear first, and next E.O.'s :

$$
H=\left(\begin{array}{c|c}
H^{\mathrm{VV}} & H^{\mathrm{VE}} \\
\hline H^{\mathrm{EV}} & H^{\mathrm{EE}}
\end{array}\right)
$$

$H^{\mathrm{vv}}$ is a $(N n \times N n)$ matrix which describes interaction between V.O.'s; $H^{\mathrm{EE}}$ is a $(\mathrm{Mm} \times \mathrm{Mm})$ matrix for the interaction between E.O.'s; $H^{\mathrm{VE}}$ is a $(\mathrm{Nn} \times \mathrm{Mm})$ matrix for the interaction between V.O.'s and E.O.'s, and $H^{\mathrm{EV}}=\left(H^{\mathrm{VE}}\right)^{+}\left({ }^{1}\right)$.

(i) Vertex-Vertex Hamiltonian $H^{\mathrm{vv}}$. - It will be understood that all $N$ V.O.'s belonging to a given type are taken together so as to correspond to a group of $N$ consecutive rows or columns in $H$ (and hence $H^{\mathrm{vv}}$ ) ; the ordering of vertices to which these V.O.'s are associated within such a group is repeated identically for all $n$ types of V.O.'s.

$H^{\mathrm{vv}}$ is the sum of two matrices, which may be written both as a direct product [11] (noted by $\otimes$ ) of a $(n \times n)$ matrix by a $(N \times N)$ matrix $\left({ }^{2}\right)$ :

$$
H^{\mathrm{vv}}=a \otimes I^{(N)}+b \otimes C
$$

where $a$ is a $(n \times n)$ Hamiltonian which describes the interaction between the different V.O.'s associated to a given vertex, $b$ is a $(n \times n)$ matrix which describes the coupling between V.O.'s belonging to neighbouring vertices, and $C$ is the $(N \times N)$ adjacency matrix of the underlying graph G. The factorization in (3) results from the assumed equivalence of all vertices and of all edges.

(ii) Vertex-Edge Hamiltonian $H^{\mathrm{VE}}$. - We must, first, order E.O.'s as we did for V.O.'s. S.E.O.'s will be taken first, grouped together $M$ by $M$, each group corresponding to a given type; A.E.O.'s are then ordered in the same way, after an (arbitrary) orientation has been chosen for all edges as explained

( $\left.{ }^{1}\right) A^{+}$, for any matrix $A$, will stand for the adjoint of $A$.

$\left({ }^{2}\right) I^{(p)}$, for any integer $p$, will stand for the unit $(p \times p)$ matrix. above. Within all groups of E.O.'s, the $M$ edges will be taken in the same order.

Together with the adjacency matrix $C$, the underlying graph $\mathrm{G}$ can be described using two vertex-edge, $(N \times M)$ matrices :

$-\Gamma_{\mathrm{S}}$ : all its elements are zero except at the intersection of a row associated with some vertex and of a column associated to an edge admitting this vertex as an extremity, the matrix element being then equal to 1 ;

- $\Gamma_{\mathrm{A}}$ : same as $\Gamma_{\mathrm{S}}$ except that non zero matrix elements are $(+1)$ when the vertex is the starting point of the oriented edge, and $(-1)$ when at its end point.

(These matrices, with different notations, have already been considered, in the same context, in ref. [7] ; they are well known in network analysis [12.])

$H^{\mathrm{VE}}$ should clearly be divided into two submatrices, for vertex-S.E.O. and vertex-A.E.O. interactions respectively :

$$
H^{\mathrm{VE}}=\left(H_{\mathrm{S}}^{\mathrm{VE}} \mid H_{\mathrm{A}}^{\mathrm{VE}}\right) .
$$

Again these matrices can be expressed as direct products :

$$
\begin{aligned}
& H_{\mathrm{S}}^{\mathrm{VE}}=c_{\mathrm{S}} \otimes \Gamma_{\mathrm{S}} \\
& H_{\mathbf{A}}^{\mathrm{VE}}=c_{\mathrm{A}} \otimes \Gamma_{\mathrm{A}}
\end{aligned}
$$

$c_{\mathrm{S}}$ and $c_{\mathrm{A}}$ are respectively $\left(n \times m_{\mathrm{S}}\right)$ and $\left(n \times m_{\mathrm{A}}\right)$ matrices describing the interaction of V.O.'s belonging to a given vertex with S.E.O.'s and A.E.O.'s belonging to edges adjacent to this vertex. $\Gamma_{\mathrm{A}}$ appears for A.E.O.'s instead of $\Gamma_{\mathrm{S}}$, because of an obvious change of sign for these orbitals, depending on the edge extremity from which they are considered.

(iii) Edge-Edge Hamiltonian $H^{\mathrm{EE}}$. - Distinguishing between S.E.O.'s and A.E.O.'s brings about an obvious submatrix decomposition :

$$
H^{\mathrm{EE}}=\left(\begin{array}{c|c}
H_{\mathrm{SS}}^{\mathrm{EE}} & H_{\mathrm{SA}}^{\mathrm{EE}} \\
\hline H_{\mathrm{AS}}^{\mathrm{EE}} & H_{\mathrm{AA}}^{\mathrm{EE}}
\end{array}\right)
$$

with

$$
\left.\begin{array}{l}
H_{\mathrm{SS}}^{\mathrm{EE}}=\left(d_{\mathrm{S}}-2 f_{\mathrm{S}}\right) \otimes I^{(M)}+f_{\mathrm{S}} \otimes \Gamma_{\mathrm{S}}^{+} \Gamma_{\mathrm{S}} \\
H_{\mathrm{AA}}^{\mathrm{EE}}=\left(d_{\mathrm{A}}-2 f_{\mathrm{A}}\right) \otimes I^{(M)}+f_{\mathrm{A}} \otimes \Gamma_{\mathbf{A}}^{+} \Gamma_{\mathbf{A}} \\
H_{\mathrm{SA}}^{\mathrm{EE}}=f_{\mathrm{SA}} \otimes \Gamma_{\mathrm{S}}^{+} \Gamma_{\mathrm{A}} \\
H_{\mathrm{AS}}^{\mathrm{EE}}=\left(H_{\mathrm{S} \mathrm{A}}^{\mathrm{EE}}\right)^{+}
\end{array}\right\}
$$

The occurrence of products such as $\Gamma_{\mathrm{S}}^{+} \Gamma_{\mathrm{S}}, \Gamma_{\mathrm{A}}^{+} \Gamma_{\mathrm{A}}$, or $\Gamma_{\mathrm{S}}^{+} \Gamma_{\mathrm{A}}$ corresponds to the fact that edge orbitals can here interact only when they belong to edges sharing a common vertex : the non-diagonal matrix elements of these products are actually different from zero (and equal to \pm 1 ) only when this condition is realized, which thus provides a convenient algebraic transcription of the topological prescription. (Dia- 
gonal matrix elements also appear in $\Gamma_{\mathrm{s}}^{+} \Gamma_{\mathrm{s}}$ and $\Gamma_{\mathrm{A}}^{+} \Gamma_{\mathrm{A}}$ and have thus to be cancelled by suitable $I^{(M)}$ terms.)

$d_{\mathbf{S}}\left(f_{\mathbf{S}}\right), \quad d_{\mathbf{A}}\left(f_{\mathbf{A}}\right), \quad f_{\mathbf{S A}}$ are respectively $\left(m_{\mathbf{S}} \times m_{\mathbf{S}}\right)$, $\left(m_{\mathbf{A}} \times m_{\mathrm{A}}\right),\left(m_{\mathbf{S}} \times m_{\mathbf{A}}\right)$ matrices. They describe the coupling between S.E.O.'s belonging to the same edge $\left(d_{\mathrm{S}}\right)$, A.E.O.'s belonging to the same edge $\left(d_{\mathrm{A}}\right)$, S.E.O.'s or A.E.O.'s belonging to neighbouring edges ( $f_{\mathrm{s}}$ and $f_{\mathrm{A}}$ respectively), S.E.O.'s from one edge and A.E.O.'s from a neighbouring edge $\left(f_{\mathrm{SA}}\right)$.

To sum up, the tight-binding Hamiltonian has the form :

$$
H=\left(\begin{array}{l|l|l}
a \otimes I^{(N)}+b \otimes C & c_{\mathrm{S}} \otimes \Gamma_{\mathrm{S}} & c_{\mathrm{A}} \otimes \Gamma_{\mathrm{A}} \\
\hline c_{\mathrm{S}}^{+} \otimes \Gamma_{\mathrm{S}}^{+} & g_{\mathrm{S}} \otimes I^{(M)}+f_{\mathrm{S}} \otimes \Gamma_{\mathrm{S}}^{+} \Gamma_{\mathrm{S}} & f_{\mathrm{SA}} \otimes \Gamma_{\mathrm{S}}^{+} \Gamma_{\mathrm{A}} \\
\hline c_{\mathrm{A}}^{+} \otimes \Gamma_{\mathrm{A}}^{+} & f_{\mathrm{SA}}^{+} \otimes \Gamma_{\mathrm{A}}^{+} \Gamma_{\mathrm{S}} & g_{\mathrm{A}} \otimes I^{(M)}+f_{\mathrm{A}} \otimes \Gamma_{\mathrm{A}}^{+} \Gamma_{\mathrm{A}}
\end{array}\right)
$$

where :

$$
\begin{aligned}
& g_{\mathrm{S}}=d_{\mathrm{S}}-2 f_{\mathrm{S}} \\
& g_{\mathrm{A}}=d_{\mathrm{A}}-2 f_{\mathrm{A}} .
\end{aligned}
$$

3. Diagonalization of the Hamiltonian. - The following relations $[7,12]$ :

$$
\left.\begin{array}{l}
\Gamma_{\mathrm{s}} \Gamma_{\mathrm{s}}^{+}=Z I^{(N)}+C \\
\Gamma_{\mathrm{A}} \Gamma_{\mathrm{A}}^{+}=Z I^{(N)}-C
\end{array}\right\}
$$

imply that both $\Gamma_{\mathrm{s}} \Gamma_{\mathrm{s}}^{+}$and $\Gamma_{\mathrm{A}} \Gamma_{\mathrm{A}}^{+}$commute with $C$. We may thus find a $(N \times N)$ unitary matrix $U$, such that

$$
\left.\begin{array}{l}
U^{+} C U=E \\
U^{+} \Gamma_{\mathrm{S}} \Gamma_{\mathrm{S}}^{+} U=F_{\mathrm{S}}=Z I^{(N)}+E \\
U^{+} \Gamma_{\mathrm{A}} \Gamma_{\mathrm{A}}^{+} U=F_{\mathrm{A}}=Z I^{(N)}-E
\end{array}\right\}
$$

where $E, F_{\mathrm{S}}, F_{\mathrm{A}}$ are $(N \times N)$ diagonal matrices.

$\Gamma_{\mathrm{S}}$ and $\Gamma_{\mathrm{A}}$ are both $(N \times M)$ matrices, with $M>N$.

Let

$$
P=M-N .
$$

There exist $P$ independent $M$-dimensional vectors which $\Gamma_{\mathrm{S}}$ sends into the null vector, and another analogous set for which $\Gamma_{\mathrm{A}}$ has the same property. Orthonormalizing and gathering together these vectors as column-vectors of two $(M \times P)$ matrices $V_{\mathrm{S}}$ and $V_{\mathrm{A}}$, we may write :

$$
\left.\begin{array}{l}
\Gamma_{\mathrm{S}} V_{\mathrm{S}}=0 \\
\Gamma_{\mathrm{A}} V_{\mathrm{A}}=0 \\
V_{\mathrm{S}}^{+} V_{\mathrm{S}}=I^{(P)} \\
V_{\mathrm{A}}^{+} V_{\mathrm{A}}=I^{(P)}
\end{array}\right\}
$$

The column-vectors of $V_{\mathrm{S}}$ (respectively $V_{\mathrm{A}}$ ) constitute a basis for a $P$-dimensional subspace of the $M$-dimensional space on which matrices $\Gamma_{\mathrm{S}}$ (respectively $\Gamma_{\mathrm{A}}$ ) are acting. The $N$-dimensional complementary subspace may be also given an orthonormal basis, the vectors of which may again be grouped as the column vectors of a $(M \times N)$ matrix $W_{\mathrm{S}}($ respectively $\left.W_{\mathrm{A}}\right)$ :

$$
\left.\begin{array}{l}
W_{\mathrm{S}}^{+} W_{\mathrm{S}}=W_{\mathrm{A}}^{+} W_{\mathrm{A}}=I^{(N)} \\
W_{\mathrm{S}}^{+} V_{\mathrm{S}}=0 \\
W_{\mathrm{A}}^{+} V_{\mathrm{A}}=0
\end{array}\right\}
$$

We then transform the Hamiltonian $H(9)$ into

$$
H^{\prime}=S^{+} H S
$$

with :

$$
S=\left(\begin{array}{c|c|c|c:c}
I^{(n)} \otimes U & 0 & 0 & 0 & 0 \\
\hline 0 & I^{\left(m_{\mathbf{S}}\right)} \otimes W_{\mathrm{S}} & I^{\left(m_{\mathbf{S}}\right)} \otimes V_{\mathrm{S}} & 0 & 0 \\
\hline 0 & 0 & 0 & I^{\left(m_{\mathbf{A}}\right)} \otimes W_{\mathrm{A}} & I^{\left(m_{\mathbf{A}}\right)} \otimes V_{\mathrm{A}}
\end{array}\right) .
$$

The unitary transformation (17) is readily accomplished using the fundamental relation connecting the usual and direct products of matrices

$$
(\alpha \otimes \beta) .(\gamma \otimes \delta)=(\alpha \cdot \gamma) \otimes(\beta . \delta)
$$

for any set of four matrices $\alpha, \beta, \gamma, \delta$ with suitable dimensions. $H^{\prime}$ is found to have the form

$$
H^{\prime}=\left(\begin{array}{c|c|c|c|c}
a \otimes I^{(N)}+b \otimes E & c_{\mathrm{S}} \otimes U^{+} \Gamma_{\mathrm{S}} W_{\mathrm{S}} & 0 & c_{\mathrm{A}} \otimes U^{+} \Gamma_{\mathrm{A}} W_{\mathrm{A}} & 0 \\
\hline c_{\mathrm{S}}^{+} \otimes W_{\mathrm{S}}^{+} \Gamma_{\mathrm{S}}^{+} U & g_{\mathrm{S}} \otimes I^{(N)}+f_{\mathrm{S}} \otimes W_{\mathrm{S}}^{+} \Gamma_{\mathrm{S}}^{+} \Gamma_{\mathrm{S}} W_{\mathrm{S}} & 0 & f_{\mathrm{SA}} \otimes W_{\mathrm{S}}^{+} \Gamma_{\mathrm{S}}^{+} \Gamma_{\mathrm{A}} W_{\mathrm{A}} & 0 \\
\hline 0 & 0 & g_{\mathrm{S}} \otimes I^{(P)} & 0 & 0 \\
\hline c_{\mathrm{A}}^{+} \otimes W_{\mathrm{A}}^{+} \Gamma_{\mathrm{A}}^{+} U & f_{\mathrm{SA}}^{+} \otimes W_{\mathrm{A}}^{+} \Gamma_{\mathrm{A}}^{+} \Gamma_{\mathrm{S}} W_{\mathrm{S}} & 0 & g_{\mathrm{A}} \otimes I^{(N)}+f_{\mathrm{A}} \otimes W_{\mathrm{A}}^{+} \Gamma_{\mathrm{A}}^{+} \Gamma_{\mathrm{A}} W_{\mathrm{A}} & 0 \\
\hline 0 & 0 & 0 & 0 & g_{\mathrm{A}} \otimes I^{(P)}
\end{array}\right) .
$$


We may immediately notice that a first diagonalization appears : $\left(m_{\mathrm{S}} P\right)$ levels and $\left(m_{\mathrm{A}} P\right)$ levels withdraw from the rest of the spectrum. They correspond respectively to a $P$-fold repetition of the $m_{\mathrm{S}}$ and $m_{\mathrm{A}}$ eigenvalues of matrices $g_{\mathrm{S}}$ and $g_{\mathrm{A}}$. These degenerate levels constitute a generalization of the $\delta$-peaks in the electronic density of states first noticed, for a simpler situation, by Weaire and Thorpe $[1,2]$.

Henceforth, we may restrict ourselves to a simpler Hamiltonian $\hat{H}$ derived from $H^{\prime}$ by suppressing the 3 rd and 5th rows and columns in (20). The encouraging feature of $\hat{H}$ consists in the appearance of submatrices like $\left(a \otimes I^{(N)}+b \otimes E\right)$, where the second factor in the direct products is a diagonal $(N \times N)$ matrix. Clearly, if all submatrices in $\hat{H}$ shared the same property, $\hat{H}$ could be factorized into $N$ square matrices of dimension $\left(n+m_{\mathrm{S}}+m_{\mathrm{A}}\right)$. To obtain this result, we shall profit by the flexibility we are left with, concerning the column vectors of matrices $W_{\mathrm{S}}$ and $W_{\mathrm{A}}$, which are only submitted to the conditions (16) of defining an orthonormal basis in some vector subspace.
In order to put, for example, the non-diagonal submatrices in the first row of (20) into the proper form, we could try, referring to (12), to take $W_{\mathrm{S}}$ and $W_{\mathrm{A}}$ in the form :

$$
\left.\begin{array}{l}
W_{\mathrm{S}}=\Gamma_{\mathrm{s}}^{+} U G_{\mathrm{S}} \\
W_{\mathrm{A}}=\Gamma_{\mathrm{A}}^{+} U G_{\mathrm{A}}
\end{array}\right\}
$$

where $G_{\mathrm{S}}$ and $G_{\mathrm{A}}$ are two diagonal $(N \times N)$ matrices. Thanks to (14), $W_{\mathrm{S}}$ and $W_{\mathrm{A}}$ thus defined are orthogonal to $V_{\mathrm{S}}$ and $V_{\mathrm{A}}$ matrices respectively, as required by the second set of relations (16). Orthonormalization of (21) yields :

$$
G_{\mathrm{S}}=\left(F_{\mathrm{S}}\right)^{-1 / 2}, \quad G_{\mathrm{A}}=\left(F_{\mathrm{A}}\right)^{-1 / 2}
$$

and the $W$ matrices take the final form :

$$
\left.\begin{array}{l}
W_{\mathrm{S}}=\Gamma_{\mathrm{S}}^{+} U\left(F_{\mathrm{S}}\right)^{-1 / 2} \\
W_{\mathrm{A}}=\Gamma_{\mathrm{A}}^{+} U\left(F_{\mathrm{A}}\right)^{-1 / 2}
\end{array}\right\}
$$

The Hamiltonian $\hat{H}$ now becomes :

$$
\hat{H}=\left(\begin{array}{l|l|l}
a \otimes I^{(N)}+b \otimes E & c_{\mathrm{S}} \otimes\left(F_{\mathrm{S}}\right)^{1 / 2} & c_{\mathrm{A}} \otimes\left(F_{\mathrm{A}}\right)^{1 / 2} \\
\hline c_{\mathrm{S}}^{+} \otimes\left(F_{\mathrm{S}}\right)^{1 / 2} & g_{\mathrm{S}} \otimes I^{(N)}+f_{\mathrm{S}} \otimes F_{\mathrm{S}} & f_{\mathrm{SA}} \otimes\left(F_{\mathrm{S}} F_{\mathrm{A}}\right)^{1 / 2} \\
\hline c_{\mathrm{A}}^{+} \otimes\left(F_{\mathrm{A}}\right)^{1 / 2} & f_{\mathrm{SA}}^{+} \otimes\left(F_{\mathrm{A}} F_{\mathrm{S}}\right)^{1 / 2} & g_{\mathrm{A}} \otimes I^{(N)}+f_{\mathrm{A}} \otimes F_{\mathrm{A}}
\end{array}\right)
$$

where all second factors in the direct products are $(N \times N)$ diagonal matrices $\left({ }^{3}\right)$. Thus, coupling only appears between rows and columns having the same index $p$ in the various submatrices of (24), and corresponding to a given eigenvalue $\varepsilon_{p}$ of $C(1 \leqslant p \leqslant N)$. To $\varepsilon_{p}$ correspond matrix elements $\left(Z+\varepsilon_{p}\right)$ and $\left(Z-\varepsilon_{p}\right)$ for $F_{\mathrm{S}}$ and $F_{\mathrm{A}},\left(Z+\varepsilon_{p}\right)^{1 / 2}$ and $\left(Z-\varepsilon_{p}\right)^{1 / 2}$ for $\left(F_{\mathrm{S}}\right)^{1 / 2}$ and $\left(F_{\mathrm{A}}\right)^{1 / 2}$.

Reordering rows and columns in (24), so that the $\left(n+m_{\mathrm{S}}+m_{\mathrm{A}}\right)$ states associated with a given eigenvalue $\varepsilon_{p}$ of the adjacency matrix $C$ be brought together, we may factorize $\hat{H}$ into a succession of $N$ subhamiltonians $h_{p}$ with

$$
h_{p}=\left(\begin{array}{l|l|l}
a+b \varepsilon_{p} & c_{\mathrm{S}}\left(Z+\varepsilon_{p}\right)^{1 / 2} & c_{\mathrm{A}}\left(Z-\varepsilon_{p}\right)^{1 / 2} \\
\hline c_{\mathrm{S}}^{+}\left(Z+\varepsilon_{p}\right)^{1 / 2} & g_{\mathrm{S}}+f_{\mathrm{S}}\left(Z+\varepsilon_{p}\right) & f_{\mathrm{SA}}\left(Z^{2}-\varepsilon_{p}^{2}\right)^{1 / 2} \\
\hline c_{\mathrm{A}}^{+}\left(Z-\varepsilon_{p}\right)^{1 / 2} & f_{\mathrm{SA}}^{+}\left(Z^{2}-\varepsilon_{p}^{2}\right)^{1 / 2} & g_{\mathrm{A}}+f_{\mathrm{A}}\left(Z-\varepsilon_{p}\right)
\end{array}\right) .
$$

Let us sum up the results obtained thus far. The tight-binding Hamiltonian (9) has :

- two sets of $P$-fold degenerate eigenvalues (where $P$ is the difference between the edge number $M$ and the vertex number $N$ ); these eigenvalues are those of the $\left(m_{\mathbf{S}} \times m_{\mathrm{S}}\right)$ submatrix $g_{\mathrm{S}}$ (which involves the interaction between bonding - actually symmetrical orbitals either within one edge, or belonging to adjacent edges), and those of the $\left(m_{\mathrm{A}} \times m_{\mathrm{A}}\right)$ submatrix $g_{\mathrm{A}}$ (which plays the same role for antibonding - more precisely antisymmetrical - orbitals);

$\left({ }^{3}\right)$ Since the eigenvalues of $C$ are confined to the interval $(-Z,+Z)$, the matrix elements of $F_{\mathrm{S}}$ and $F_{\mathrm{A}}$ are real positive, so that $\left(F_{\mathrm{S}}\right)^{1 / 2}$ and $\left(F_{\mathrm{A}}\right)^{1 / 2}$ are real and equal to their adjoint.
$-\left(n+m_{\mathrm{S}}+m_{\mathrm{A}}\right)$ energy bands each one with $N$ levels; these can be obtained by diagonalizing the $N$ subhamiltonians (25).

Thus the diagonalization of $H$ involves three steps :

(i) diagonalization of matrices $g_{\mathrm{S}}$ and $g_{\mathrm{A}}$, which gives the $\delta$-peak contributions to the density of states;

(ii) diagonalization of the adjacency matrix $C$; (only eigenvalues are necessary and no information on the eigenvectors is required) ;

(iii) for every eigenvalue $\varepsilon_{p}$ of $C$, diagonalization of the corresponding subhamiltonian (25). This is of course highly reminiscent of the Bloch transformation for a periodic system, where the initial Hamiltonian may be factorized into $k$-dependent subhamil- 
tonians. A considerable reduction in computational difficulty results in both cases.

This factorization generalizes the transformation of a «4-band " Hamiltonian into a "1-band " Hamiltonian, proposed by Weaire and Thorpe for 4-fold coordinated covalent semiconductors.

\section{Systems with two types of vertices and a bipartite} graph. - 4.1 HAMILTONIAN STRUCTURE. - The general ideas underlying the previous treatment remain unchanged. But now, the graph vertices fall into two classes, labelled 1 and 2 respectively, and the graph $\mathrm{G}$ only links vertices belonging to different types. All vertices of type 1 are identical, with degree $Z_{1}$, and all vertices of type 2 are also similar one to the other, with degree $Z_{2}$. All edges, from a 1-vertex to a 2-vertex, are also identical. If $N_{1}$, $N_{2}$ and $M$ are the respective numbers of 1-vertices, 2 -vertices and edges, the following relations hold :

$$
Z_{1} N_{1}=Z_{2} N_{2}=M
$$

$Z_{1}$ and $Z_{2}$ are taken to be strictly greater than 2 , so that

$$
M>N_{1}+N_{2}=N
$$

We shall assume $Z_{1} \geqslant Z_{2}$, so that :

$$
Q=N_{2}-N_{1} \geqslant 0 \text {. }
$$

Putting together 1-vertices first gives the graph adjacency matrix $C$ the form

$$
C=\left(\begin{array}{c|c}
0 & B \\
\hline B^{+} & 0
\end{array}\right)
$$

where $B$ is the $\left(N_{1} \times N_{2}\right)$ matrix describing the linking of 1 -vertices to 2 -vertices. In the same way, the $\Gamma_{\mathrm{s}}$ matrix of the previous treatment now decomposes into

$$
\Gamma_{\mathrm{S}}=\left(\frac{\Gamma_{1}}{\Gamma_{2}}\right)
$$

where $\Gamma_{1}$ and $\Gamma_{2}$ are respectively $\left(N_{1} \times M\right)$ and $\left(N_{2} \times M\right)$ matrices. The following relations hold :

$$
\left.\begin{array}{l}
\Gamma_{1} \Gamma_{1}^{+}=Z_{1} I^{\left(N_{1}\right)} \\
\Gamma_{2} \Gamma_{2}^{+}=Z_{2} I^{\left(N_{2}\right)} \\
\Gamma_{1} \Gamma_{2}^{+}=B
\end{array}\right\}
$$

Atomic orbitals will again be divided into two categories :

- vertex orbitals (V.O.), distinguishing of course 1and 2-vertices, with respectively, $n_{1}$ and $n_{2}$ V.O.'s per vertex,

- edge orbitals (E.O.). However, edges are not now symmetrical and the $m$ E.O.'s of a given edge cannot be characterized as symmetrical or antisymmetrical.

The tight-binding hamiltonian $H$ keeps its general form (2), but, now, the vertex-vertex Hamiltonian $H^{\mathrm{vv}}$ decomposes into

$$
H^{\mathrm{vv}}=\left(\begin{array}{l|l}
a_{1} \otimes I^{\left(N_{1}\right)} & b \otimes B \\
\hline b^{+} \otimes B^{+} & a_{2} \otimes I^{\left(N_{2}\right)}
\end{array}\right)
$$

where $a_{1}$ and $a_{2}$ are respectively $\left(n_{1} \times n_{1}\right)$ and $\left(n_{2} \times n_{2}\right)$ matrices describing the V.O.'s interactions within a 1 -vertex or a 2-vertex, and $b$ is a $\left(n_{1} \times n_{2}\right)$ matrix for the coupling between V.O.'s on adjacent vertices.

The vertex-edge hamiltonian $H^{\mathrm{VE}}$ takes the form

$$
H^{\mathrm{VE}}=\left(\frac{c_{1} \otimes \Gamma_{1}}{c_{2} \otimes \Gamma_{2}}\right)
$$

where $c_{1}$ and $c_{2}$ are respectively $\left(n_{1} \times m\right)$ and $\left(n_{2} \times m\right)$ matrices describing the interactions between V.O.'s and E.O.'s on adjacent vertices and edges.

Finally, the edge-edge Hamiltonian $H^{\mathrm{EE}}$ may be written as a sum of direct products, essentially as in (8):

$$
\begin{aligned}
H^{\mathrm{EE}}=\left(d-f_{1}\right. & \left.-f_{2}\right) \otimes I^{(M)}+ \\
& +f_{1} \otimes \Gamma_{1}^{+} \Gamma_{1}+f_{2} \otimes \Gamma_{2}^{+} \Gamma_{2}
\end{aligned}
$$

where the $(m \times m)$ matrices $d, f_{1}, f_{2}$ describe the interactions between E.O.'s belonging respectively to the same edge, to edges adjacent to the same 1 -vertex and to edges adjacent to the same 2-vertex. In the following we shall put :

$$
g=d-f_{1}-f_{2} .
$$

The whole initial Hamiltonian is thus :

$$
H=\left(\begin{array}{l|l|l}
a_{1} \otimes I^{\left(N_{1}\right)} & b \otimes B & c_{1} \otimes \Gamma_{1} \\
\hline b^{+} \otimes B^{+} & a_{2} \otimes I^{\left(N_{2}\right)} & c_{2} \otimes \Gamma_{2} \\
\hline c_{1}^{+} \otimes \Gamma_{1}^{+} & c_{2}^{+} \otimes \Gamma_{2}^{+} & g \otimes I^{(M)}+f_{1} \otimes \Gamma_{1}^{+} \Gamma_{1}+f_{2} \otimes \Gamma_{2}^{+} \Gamma_{2}
\end{array}\right) .
$$

4.2 Diagonalization of the Hamiltonian. The main purpose of the following calculation will be, as before, to devise a suitable unitary transformation which will transform (36) into a matrix, the sub- 
matrices of which are all simultaneously diagonal. In the previous case, the condition for such a transformation was the fact that, owing to (11), $C$ and $\left(\Gamma_{\mathrm{S}} \Gamma_{\mathrm{S}}^{+}\right)$ commuted.
In the present case a similar diagonalization is possible thanks to (31). The proper unitary transformation of $H$ involves the unitary matrix (analogous to (18)) :

$$
S=\left(\begin{array}{l|l|l|l|l}
I^{\left(n_{1}\right)} \otimes U_{1} & & & & \\
\hline & I^{\left(n_{2}\right)} \otimes U_{2} & I^{\left(n_{2}\right)} \otimes U_{3} & & \\
\hline & & & I^{(m)} \otimes W & I^{(m)} \otimes V
\end{array}\right)
$$

where :

(a) $U_{1}$ is a unitary $\left(N_{1} \times N_{1}\right)$ matrix diagonalizing $\left(B B^{+}\right)$:

$$
\left.\begin{array}{rl}
U_{1}^{+} U_{1} & =I^{\left(N_{1}\right)} \\
U_{1}^{+} B B^{+} U_{1} & =D
\end{array}\right\}
$$

( $D$ is a diagonal matrix with positive elements) ;

(b) $U_{2}$ is a $\left(N_{2} \times N_{1}\right)$ matrix with

$$
U_{2}=B^{+} U_{1} D^{-1 / 2}
$$

and thus :

$$
\left.\begin{array}{rl}
U_{2}^{+} U_{2} & =I^{\left(N_{1}\right)} \\
U_{1}^{+} B U_{2} & =D^{1 / 2} \equiv E
\end{array}\right\}
$$

$E$ is a diagonal matrix, the eigenvalues $\varepsilon_{p}$ of which will be taken $>0$. The eigenvalues of $C$ (29) are obviously $\left( \pm \varepsilon_{p}\right)$ with $1 \leqslant p \leqslant N_{1}$, and 0 with a degeneracy of order $Q$;

(c) $U_{3}$ is a $\left(N_{2} \times Q\right)$ matrix such that

$$
\left.\begin{array}{rl}
U_{3}^{+} U_{3} & =I^{(Q)} \\
B U_{3} & =0 \\
U_{2}^{+} U_{3} & =0
\end{array}\right\}
$$

(d) $V$ is a $(M \times P)$ matrix $(P=M-N)$, with

$$
\left.\begin{array}{l}
\Gamma_{1} V=0 ; \quad \Gamma_{2} V=0 \\
V^{+} V=I^{(P)}
\end{array}\right\} .
$$

(e) $W$ is a $(M \times N)$ matrix with

$$
\begin{aligned}
& W^{+} V=0 \\
& W^{+} W=I^{(N)}
\end{aligned}
$$

$H$ becomes

$H^{\prime}=S^{+} H S=\left(\begin{array}{c|c|c|c|c}\frac{a_{1} \otimes I^{\left(N_{1}\right)}}{b^{+} \otimes E} & b \otimes E & 0 & c_{1} \otimes U_{1}^{+} \Gamma_{1} W & 0 \\ \hline 0 & a_{2} \otimes I^{\left(N_{1}\right)} & 0 & c_{2} \otimes U_{2}^{+} \Gamma_{2} W & 0 \\ \hline c_{1}^{+} \otimes W^{+} \Gamma_{1}^{+} U_{1} & 0 & a_{2} \otimes I^{(Q)} & c_{2} \otimes U_{3}^{+} \Gamma_{2} W & 0 \\ \hline 0 & c_{2}^{+} \otimes W^{+} \Gamma_{2}^{+} U_{2} & c_{2}^{+} \otimes W^{+} \Gamma_{2}^{+} U_{3} & \begin{array}{c}g \otimes I^{(N)}+ \\ +f_{1} \otimes W^{+} \Gamma_{1}^{+} \Gamma_{1} W \\ +f_{2} \otimes W^{+} \Gamma_{2}^{+} \Gamma_{2} W\end{array} & 0 \\ \hline 0 & 0 & 0 & g \otimes I^{(P)}\end{array}\right)$

We notice that $m$ edge levels - each of which is $P$-fold degenerate - separate from the spectrum as in the previous case. In the remaining Hamiltonian $\hat{H}$, the four submatrices in the upper left corner already show the proper diagonal form. There remains the $W$ matrix to define, in order to bring further diagonalization in the submatrices of the 4 th row and column of (45).

Let us take $W$ as :

with :

$$
W=\left(W_{1}\left|W_{2}\right| W_{3}\right) \cdot R
$$

$$
\begin{aligned}
& W_{1}=\Gamma_{1}^{+} U_{1} \\
& \left.W_{2}=\Gamma_{2}^{+} U_{2}\right\} \text {. } \\
& W_{3}=\Gamma_{2}^{+} U_{3}
\end{aligned}
$$

Thanks to (38)-(42), condition (43) is satisfied, and (44) becomes

$$
R^{+} \cdot\left(\begin{array}{c|c|c}
Z_{1} I^{\left(N_{1}\right)} & E & 0 \\
\hline E & Z_{2} I^{\left(N_{2}\right)} & 0 \\
\hline 0 & 0 & Z_{2} I^{(Q)}
\end{array}\right) \cdot R=I^{(N)}
$$


$R$ can be determined up to a rotation in $N$-space. This matrix may be taken as made up of properly defined $\left(N_{1} \times N_{1}\right)$ (and $(Q \times Q)$ for $\left.L\right)$ diagonal submatrices:

$$
R=\left(\begin{array}{c|c|c}
J_{1} & K_{1} & 0 \\
\hline J_{2} & K_{2} & 0 \\
\hline 0 & 0 & L
\end{array}\right) .
$$

A simple possible choice is $\left({ }^{4}\right)$ :

$$
\begin{aligned}
J_{1} & =\frac{1}{2}\left(P_{1}\right)^{-1 / 2} & P_{1} & =\frac{1}{2} \sqrt{\frac{Z_{1}}{Z_{2}}}\left(\sqrt{Z_{1} Z_{2}} I^{\left(N_{1}\right)}+E\right) \\
J_{2} & =\frac{1}{2}\left(P_{2}\right)^{-1 / 2} & P_{2} & =\frac{1}{2} \sqrt{\frac{Z_{2}}{Z_{1}}}\left(\sqrt{Z_{1} Z_{2}} I^{\left(N_{1}\right)}+E\right) \\
K_{1} & =\frac{1}{2}\left(Q_{1}\right)^{-1 / 2} & Q_{1} & =\frac{1}{2} \sqrt{\frac{Z_{1}}{Z_{2}}}\left(\sqrt{Z_{1} Z_{2}} I^{\left(N_{1}\right)}-E\right) \\
K_{2} & =-\frac{1}{2}\left(Q_{2}\right)^{-1 / 2} & Q_{2} & =\frac{1}{2} \sqrt{\frac{Z_{2}}{Z_{1}}}\left(\sqrt{Z_{1} Z_{2}} I^{\left(N_{1}\right)}-E\right) \\
L & =\left(Z_{2}\right)^{-1 / 2} I^{(Q)} & &
\end{aligned}
$$

the final form of $\hat{H}$ is :

$\hat{H}=\left(\begin{array}{c|l|c|l|l|c}a_{1} \otimes I^{\left(N_{1}\right)} & h \otimes E & 0 & c_{1} \otimes\left(P_{1}\right)^{1 / 2} & c_{1} \otimes\left(Q_{1}\right)^{1 / 2} & 0 \\ \hline h^{+} \otimes E & a_{2} \otimes I^{\left(N_{1}\right)} & 0 & c_{2} \otimes\left(P_{2}\right)^{1 / 2} & -c_{2} \otimes\left(Q_{2}\right)^{1 / 2} & 0 \\ \hline 0 & 0 & a_{2} \otimes I^{(Q)} & 0 & 0 & c_{2} \otimes \sqrt{Z_{2}} I^{(Q)} \\ \hline c_{1}^{+} \otimes\left(P_{1}\right)^{1 / 2} & c_{2}^{+} \otimes\left(P_{2}\right)^{1 / 2} & 0 & \begin{array}{l}g \otimes I^{\left(N_{1}\right)}+ \\ +f_{1} \otimes P_{1} \\ +f_{2} \otimes P_{2}\end{array} & \begin{array}{c}f_{1} \otimes\left(P_{1} Q_{1}\right)^{1 / 2}- \\ -f_{2} \otimes\left(P_{2} Q_{2}\right)^{1 / 2}\end{array} & 0 \\ \hline c_{1}^{+} \otimes\left(Q_{1}\right)^{1 / 2} & -c_{2}^{+} \otimes\left(Q_{2}\right)^{1 / 2} & 0 & \begin{array}{l}f_{1}^{+} \otimes\left(P_{1} Q_{1}\right)^{1 / 2}- \\ -f_{2}^{+} \otimes\left(P_{2} Q_{2}\right)^{1 / 2}\end{array} & \begin{array}{l}g \otimes I^{\left(N_{1}\right)}+ \\ +f_{1} \otimes Q_{1} \\ +f_{2} \otimes Q_{2}\end{array} & 0 \\ \hline 0 & 0 & c_{2}^{+} \otimes \sqrt{Z_{2}} I^{(Q)} & 0 & 0 & \begin{array}{l}\left(g+Z_{2} f_{2}\right) \otimes \\ \otimes I^{(Q)}\end{array}\end{array}\right)$

Eq. (51) is the equivalent of eq. (24) in the previous case.

It should be remarked that a further set of $\left(n_{2}+m\right)$ discrete levels (now with $Q$-fold degeneracy) appears ; the corresponding eigenvectors mix E.O.'s and V.O.'s from the majority vertices (here of type 2). The eigenvalues are those of $h_{0}$ given by :

$$
h_{0}=\left(\begin{array}{l|l}
a_{2} & \sqrt{Z_{2}} c_{2} \\
\hline \sqrt{Z_{2}} c_{2}^{+} & g+Z_{2} f_{2}
\end{array}\right) .
$$

In a manner quite similar to that used in the previous section, we may factorize the remaining Hamiltonian into a succession of $N_{1}$ subhamiltonians $h_{p}\left(1 \leqslant p \leqslant N_{1}\right)$ of rank $\left(n_{1}+n_{2}+2 m\right)$, each corresponding to an eigenvalue $\varepsilon_{p}$ of $E$ (or to a pair of opposite eigenvalues $\pm \varepsilon_{p}$ of the adjacency matrix $C$ ) :

$h_{p}=\left(\begin{array}{l|l|l|l}a_{1} & h \varepsilon_{p} & c_{1} \alpha_{1}\left(\bar{Z}+\varepsilon_{p}\right)^{1 / 2} & c_{1} \alpha_{1}\left(\bar{Z}-\varepsilon_{p}\right)^{1 / 2} \\ \hline h^{+} \varepsilon_{p} & a_{2} & c_{2} \alpha_{2}\left(\bar{Z}+\varepsilon_{p}\right)^{1 / 2} & -c_{2} \alpha_{2}\left(\bar{Z}-\varepsilon_{p}\right)^{1 / 2} \\ \hline c_{1}^{+} \alpha_{1}\left(\bar{Z}+\varepsilon_{p}\right)^{1 / 2} & c_{2}^{+} \alpha_{2}\left(\bar{Z}+\varepsilon_{p}\right)^{1 / 2} & g+\left(f_{1} \alpha_{1}^{2}+f_{2} \alpha_{2}^{2}\right)\left(\bar{Z}+\varepsilon_{p}\right) & \left(f_{1} \alpha_{1}^{2}-f_{2} \alpha_{2}^{2}\right)\left(\bar{Z}^{2}-\varepsilon_{p}^{2}\right)^{1 / 2} \\ \hline c_{1}^{+} \alpha_{1}\left(\bar{Z}-\varepsilon_{p}\right)^{1 / 2} & -c_{2}^{+} \alpha_{2}\left(\bar{Z}-\varepsilon_{p}\right)^{1 / 2} & \left(f_{1}^{+} \alpha_{1}^{2}-f_{2}^{+} \alpha_{2}^{2}\right)\left(\bar{Z}^{2}-\varepsilon_{p}^{2}\right)^{1 / 2} & g+\left(f_{1} \alpha_{1}^{2}+f_{2} \alpha_{2}^{2}\right)\left(\bar{Z}-\varepsilon_{p}\right)\end{array}\right)$

(4) One can show that the eigenvalues of $E$ are confined to the interval $\left(-\sqrt{Z_{1} Z_{2}},+\sqrt{Z_{1} Z_{2}}\right)$ so that the matrix elements of $J_{1}, J_{2}, K_{1}, K_{2}$ are real and these matrices are equal to their adjoint. 
where

$$
\left.\begin{array}{l}
\alpha_{1}=\frac{1}{\sqrt{2}}\left(\frac{Z_{1}}{Z_{2}}\right)^{1 / 4} \quad \alpha_{2}=\frac{1}{\sqrt{2}}\left(\frac{Z_{2}}{Z_{1}}\right)^{1 / 4} \\
\bar{Z}=\sqrt{Z_{1} Z_{2}}
\end{array}\right\} .
$$

Let us sum up the results for this second class of systems. The Hamiltonian (36) has :

- one set of $m P$-fold degenerate eigenvalues which are the eigenvalues of the edge-edge coupling matrix $g$ (35),

- one set of $\left(n_{2}+m\right) Q$-fold degenerate eigenvalues, those of $h_{0}$ given by (52),

- $N_{1}$ sets of $\left(n_{1}+n_{2}+2 m\right)$ eigenvalues obtained by diagonalizing (53) for each $\varepsilon_{p}$.

Again we have realized a diagonalization of $H$ analogous to that obtained in section $3\left(^{5}\right)$.

5. An example of application. - In order to show how the general formalism presented in the foregoing may be worked out practically, we shall now revert to the simpler example of one type of vertex and repeat the calculation of the electronic band structure of $\mathrm{GeSe}_{2}$ presented by Lannoo and Bensoussan in [10].

In this paper, a LCAO treatment is put forward, which involves the atomic orbitals shown on figure 1 :

- every $\mathrm{Ge}$ atom has 4 equivalent $\mathrm{sp}^{3}$ orbitals $\left(\sigma_{1}, \sigma_{1}^{\prime}\right.$, etc... for atom $1, \sigma_{2}$ for atom 2 etc...) ;

- every Se atom bears two p-orbitals involved in covalent bonds with the neighbouring $\mathrm{Ge}$ atoms $\left(p_{1}\right.$ and $\left.p_{2}\right)$, one lone-pair s-orbital, and another p-type lone-pair orbital labeled $\tau$.

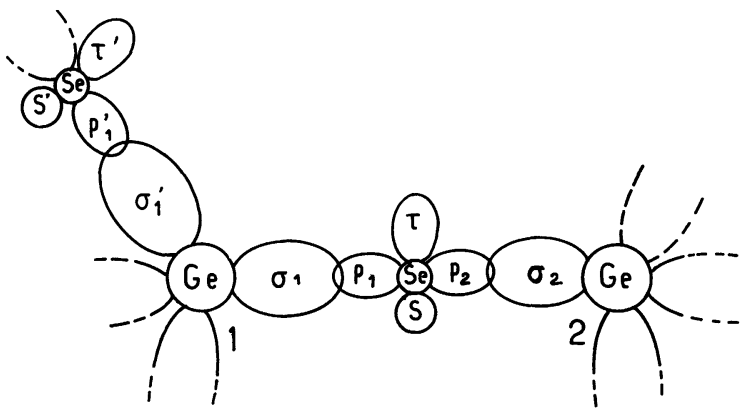

Fig. 1. - Atomic orbitals in $\mathrm{GeSe}_{2}$.

Lannoo and Bensoussan have excluded all interactions involving these $\mathrm{s}$ and $\tau$ Se-orbitals. We shall here assume the same kind of approximation in order to keep as close as possible to their treatment, for the sake of comparison. To these atomic orbitals thus correspond two $\delta$-peaks in the density of states, shown as « $\mathrm{S}$ » and « $\tau$ » on figure 2 .

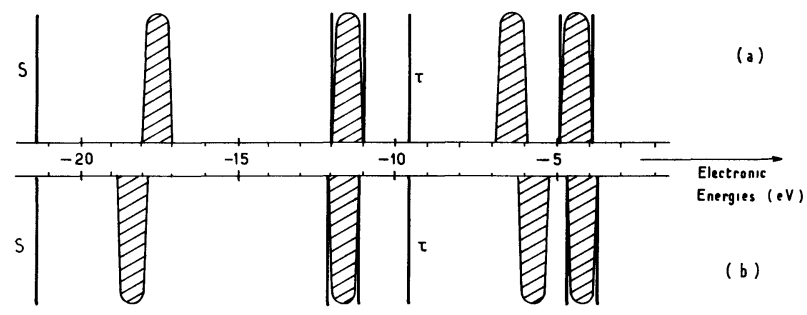

Fig. 2. - Energy bands for $\mathrm{GeSe}_{2}:(a)$ without interaction between bonding and antibonding orbitals (cf. [10]); $(b)$ with this interaction taken into account.

In the same way, we shall only retain, as in [10], the following matrix elements for the electronic Hamiltonian $H$ :

$$
\begin{array}{r}
\left\langle\sigma_{1}|H| \sigma_{1}\right\rangle=\alpha_{\mathrm{Ge}}, \quad\left\langle\mathrm{p}_{1}|H| \mathrm{p}_{1}\right\rangle=\alpha_{\mathrm{Se}}, \\
\left\langle\sigma_{1}|H| \mathrm{p}_{1}\right\rangle=\beta,
\end{array}
$$

and

$$
\left\langle\sigma_{1}|H| \sigma_{1}^{\prime}\right\rangle,\left\langle\mathrm{p}_{1}|H| \sigma_{2}\right\rangle, \quad\left\langle\mathrm{p}_{1}|H| \sigma_{1}^{\prime}\right\rangle .
$$

First, we replace the atomic orbitals along the Ge-Se bonds by their bonding and antibonding linear combinations, $\mathrm{A}_{1}$ and $\mathrm{B}_{1}$ instead of $\sigma_{1}$ and $\mathrm{p}_{1}$, with

$$
\left.\begin{array}{l}
\left|\mathrm{A}_{1}\right\rangle=b\left|\sigma_{1}\right\rangle-a\left|\mathrm{p}_{1}\right\rangle \\
\left|\mathrm{B}_{1}\right\rangle=a\left|\sigma_{1}\right\rangle+b\left|\mathrm{p}_{1}\right\rangle
\end{array}\right\},
$$

and similarly $\mathrm{A}_{2}$ and $\mathrm{B}_{2}$ instead of $\sigma_{2}, \mathrm{p}_{2}, \mathrm{~A}_{1}^{\prime}$ and $\mathrm{B}_{1}^{\prime}$ instead of $\sigma_{1}^{\prime}, \mathrm{p}_{1}^{\prime}$ etc. ; $a$ and $b$ are given by :

$$
\left.\begin{array}{l}
a^{2}+b^{2}=1 \\
\frac{b}{a}=\frac{\alpha_{\mathrm{Se}}-E_{\mathrm{A}}}{\beta}=\frac{E_{\mathrm{B}}-\alpha_{\mathrm{Ge}}}{\beta}
\end{array}\right\}
$$

and

$$
\begin{aligned}
& E_{\mathrm{A}}=\left\langle\mathrm{A}_{1}|H| \mathrm{A}_{1}\right\rangle=\frac{1}{2}\left(\alpha_{\mathrm{Se}}+\alpha_{\mathrm{Ge}}\right)+\sqrt{\frac{\left(\alpha_{\mathrm{Se}}-\alpha_{\mathrm{Ge}}\right)^{2}}{4}+\beta^{2}} \\
& \left.\begin{array}{rl}
E_{\mathrm{B}}= & \left\langle\mathrm{B}_{1}|H| \mathrm{B}_{1}\right\rangle=\frac{1}{2}\left(\alpha_{\mathrm{Se}}+\alpha_{\mathrm{Ge}}\right)-\sqrt{\frac{\left(\alpha_{\mathrm{Se}}-\alpha_{\mathrm{Ge}}\right)^{2}}{4}+\beta^{2}} \\
\left\langle\mathrm{~A}_{1}|H| \mathrm{B}_{1}\right\rangle=0
\end{array}\right\}
\end{aligned}
$$

$\left({ }^{5}\right)$ If 1- and 2-vertices are identical, one may show (by separating symmetrical and antisymmetrical orbitals) that (53) can be further factorized into two Hamiltonians, one identical to (25) and one obtained by changing in (25) the sign of $\varepsilon_{p}$. One thus recovers the results of the previous case. 
We shall also keep the numerical values used in [10], that is

$$
\left.\begin{array}{ll}
\alpha_{\mathrm{Ge}}=-8.37 \mathrm{eV}, & \alpha_{\mathrm{Se}}=-9.53 \mathrm{eV}, \quad \beta=-4.00 \mathrm{eV} \\
E_{\mathrm{A}}=-4.91 \mathrm{eV}, & E_{\mathrm{B}}=-12.99 \mathrm{eV}, \quad a b=0.495, \quad b^{2}-a^{2}=0.140
\end{array}\right\} .
$$

Bonding orbitals on the one hand, antibonding orbitals on the other, interact via the following matrix elements :

$$
\left.\begin{array}{l}
\left\langle\mathrm{A}_{1}|H| \mathrm{A}_{2}\right\rangle=-2 a b\left\langle\mathrm{p}_{1}|H| \sigma_{2}\right\rangle=\Delta_{\mathrm{A}}^{\prime}=-0.5 \mathrm{eV} \\
\left\langle\mathrm{B}_{1}|H| \mathrm{B}_{2}\right\rangle=+2 a b\left\langle\mathrm{p}_{1}|H| \sigma_{2}\right\rangle=\Delta_{\mathrm{B}}^{\prime}=+0.5 \mathrm{eV} \\
\left\langle\mathrm{A}_{1}|H| \mathrm{A}_{1}^{\prime}\right\rangle=b^{2}\left\langle\sigma_{1}|H| \sigma_{1}^{\prime}\right\rangle-2 a b\left\langle\mathrm{p}_{1}|H| \sigma_{1}^{\prime}\right\rangle=\Delta_{\mathrm{A}}=-0.5 \mathrm{eV} \\
\left\langle\mathrm{B}_{1}|H| \mathrm{B}_{1}^{\prime}\right\rangle=a^{2}\left\langle\sigma_{1}|H| \sigma_{1}^{\prime}\right\rangle+2 a b\left\langle\mathrm{p}_{1}|H| \sigma_{1}^{\prime}\right\rangle=\Delta_{\mathrm{B}}=-1.5 \mathrm{eV}
\end{array}\right\} .
$$

These are the only interactions that have been taken into account by Lannoo and Bensoussan : bonding and antibonding orbitals are not coupled together and may thus be treated separately, which brings great simplification into the problem. However, some inconsistency is brought into the picture by this approximation, since the Hamiltonian matrix elements between bonding and antibonding orbitals are a priori non zero. Indeed, we find :

$$
\left.\begin{array}{l}
\left\langle\mathrm{A}_{1}|H| \mathrm{B}_{2}\right\rangle=\left(b^{2}-a^{2}\right)\left\langle\mathrm{p}_{1}|H| \sigma_{2}\right\rangle=\Delta_{\mathrm{AB}}^{\prime} \\
\left\langle\mathrm{A}_{1}|H| \mathrm{B}_{1}^{\prime}\right\rangle=a b\left\langle\sigma_{1}|H| \sigma_{1}^{\prime}\right\rangle+\left(b^{2}-a^{2}\right)\left\langle\mathrm{p}_{1}|H| \sigma_{1}^{\prime}\right\rangle=\Delta_{\mathrm{AB}}
\end{array}\right\} .
$$

Numerically, (60) gives

$$
\left.\begin{array}{l}
\Delta_{\mathrm{AB}}^{\prime}=\frac{a^{2}-b^{2}}{2 a b} \Delta_{\mathrm{A}}^{\prime} \simeq 0.07 \mathrm{eV} \\
\Delta_{\mathrm{AB}}=a b\left(\Delta_{\mathrm{A}}+\Delta_{\mathrm{B}}\right)+\frac{b^{2}-a^{2}}{2 a b}\left(\Delta_{\mathrm{B}}-\Delta_{\mathrm{A}}\right) \simeq 1 \mathrm{eV}
\end{array}\right\} .
$$

While $\Delta_{\mathrm{AB}}^{\prime}$ is effectively negligible, $\Delta_{\mathrm{AB}}$ is of the same order of magnitude as the other matrix elements (59), and is worth taking into account. Thanks to the general formalism we have at our disposal, this can be done in a reasonably simple way, as we now shall see.

All orbitals here are bond-orbitals (no vertex-orbital). Let us introduce S.E.O.'s and A.E.O.'s such as

$$
\left.\begin{array}{ll}
\left|A_{S}\right\rangle=\frac{1}{\sqrt{2}}\left(\left|A_{1}\right\rangle+\left|A_{2}\right\rangle\right), & \left|A_{A}\right\rangle=\frac{1}{\sqrt{2}}\left(\left|A_{1}\right\rangle-\left|A_{2}\right\rangle\right) \\
\left|B_{S}\right\rangle=\frac{1}{\sqrt{2}}\left(\left|B_{1}\right\rangle+\left|B_{2}\right\rangle\right), & \left|B_{A}\right\rangle=\frac{1}{\sqrt{2}}\left(\left|B_{1}\right\rangle-\left|B_{2}\right\rangle\right)
\end{array}\right\}
$$

with matrix elements

$$
\left.\begin{array}{ll}
\left\langle\mathrm{A}_{\mathrm{S}}|H| \mathrm{A}_{\mathrm{S}}\right\rangle=E_{\mathrm{A}}+\Delta_{\mathrm{A}}^{\prime}, & \left\langle\mathrm{A}_{\mathrm{A}}|H| \mathrm{A}_{\mathrm{A}}\right\rangle=E_{\mathrm{A}}-\Delta_{\mathrm{A}}^{\prime}, \\
\left\langle\mathrm{B}_{\mathrm{S}}|H| \mathrm{B}_{\mathrm{S}}\right\rangle=E_{\mathrm{B}}+\Delta_{\mathrm{B}}^{\prime}, & \left\langle\mathrm{B}_{\mathrm{A}}|H| \mathrm{B}_{\mathrm{A}}\right\rangle=E_{\mathrm{B}}-\Delta_{\mathrm{B}}^{\prime} \\
\left\langle\mathrm{A}_{\mathrm{S}}|H| \mathrm{B}_{\mathrm{S}}\right\rangle=0, & \left\langle\mathrm{~A}_{\mathrm{A}}|H| \mathrm{B}_{\mathrm{A}}\right\rangle=0,
\end{array}\right\}
$$

between orbitals along the same Ge-Ge bond, and

$$
\begin{aligned}
& \left\langle\mathrm{A}_{\mathrm{S}}|H| \mathrm{A}_{\mathrm{S}}^{\prime}\right\rangle=\left\langle\mathrm{A}_{\mathrm{A}}|H| \mathrm{A}_{\mathrm{A}}^{\prime}\right\rangle=\left\langle\mathrm{A}_{\mathrm{S}}|H| \mathrm{A}_{\mathrm{A}}^{\prime}\right\rangle=\frac{1}{2}\left\langle\mathrm{~A}_{1}|H| \mathrm{A}_{1}^{\prime}\right\rangle=\Delta_{\mathrm{A}} / 2, \\
& \left\langle\mathrm{~B}_{\mathrm{S}}|H| \mathrm{B}_{\mathrm{S}}^{\prime}\right\rangle=\left\langle\mathrm{B}_{\mathrm{A}}|H| \mathrm{B}_{\mathrm{A}}^{\prime}\right\rangle=\left\langle\mathrm{B}_{\mathrm{S}}|H| \mathrm{B}_{\mathrm{A}}^{\prime}\right\rangle=\frac{1}{2}\left\langle\mathrm{~B}_{1}|H| \mathrm{B}_{1}^{\prime}\right\rangle=\Delta_{\mathrm{B}} / 2, \\
& \left\langle\mathrm{~A}_{\mathrm{S}}|H| \mathrm{B}_{\mathrm{S}}^{\prime}\right\rangle=\left\langle\mathrm{A}_{\mathrm{A}}|H| \mathrm{B}_{\mathrm{A}}^{\prime}\right\rangle=\left\langle\mathrm{A}_{\mathrm{S}}|H| \mathrm{B}_{\mathrm{A}}^{\prime}\right\rangle=\frac{1}{2}\left\langle\mathrm{~A}_{1}|H| \mathrm{B}_{1}^{\prime}\right\rangle=\Delta_{\mathrm{AB}} / 2, \\
& \left\langle\mathrm{~B}_{\mathrm{S}}|H| \mathrm{A}_{\mathrm{S}}^{\prime}\right\rangle=\left\langle\mathrm{B}_{\mathrm{A}}|H| \mathrm{A}_{\mathrm{A}}^{\prime}\right\rangle=\left\langle\mathrm{B}_{\mathrm{S}}|H| \mathrm{A}_{\mathrm{A}}^{\prime}\right\rangle=\frac{1}{2}\left\langle\mathrm{~B}_{1}|H| \mathrm{A}_{1}^{\prime}\right\rangle=\Delta_{\mathrm{AB}} / 2,
\end{aligned}
$$

for orbitals along two neighbouring Ge-Ge bonds adjacent to the same Ge atom. 
The submatrices of the edge-edge Hamiltonian introduced in section 2 thus read :

$$
\begin{aligned}
& d_{\mathrm{S}}=\left(\begin{array}{cc}
E_{\mathrm{A}}+\Delta_{\mathrm{A}}^{\prime} & 0 \\
0 & E_{\mathrm{B}}+\Delta_{\mathrm{B}}^{\prime}
\end{array}\right) \quad d_{\mathrm{A}}=\left(\begin{array}{cc}
E_{\mathrm{A}}-\Delta_{\mathrm{A}}^{\prime} & 0 \\
0 & E_{\mathrm{B}}-\Delta_{\mathrm{B}}^{\prime}
\end{array}\right) \\
& f_{\mathrm{S}}=f_{\mathrm{A}}=f_{\mathrm{SA}}=\frac{1}{2}\left(\begin{array}{lc}
\Delta_{\mathrm{A}} & \Delta_{\mathrm{AB}} \\
\Delta_{\mathrm{AB}} & \Delta_{\mathrm{B}}
\end{array}\right) \\
& g_{\mathrm{S}}=\left(\begin{array}{cc}
E_{\mathrm{A}}+\Delta_{\mathrm{A}}^{\prime}-\Delta_{\mathrm{A}} & -\Delta_{\mathrm{AB}} \\
-\Delta_{\mathrm{AB}} & E_{\mathrm{B}}+\Delta_{\mathrm{B}}^{\prime}-\Delta_{\mathrm{B}}
\end{array}\right) \\
& g_{\mathrm{A}}=\left(\begin{array}{cc}
E_{\mathrm{A}}-\Delta_{\mathrm{A}}^{\prime}-\Delta_{\mathrm{A}} & -\Delta_{\mathrm{AB}} \\
-\Delta_{\mathrm{AB}} & E_{\mathrm{B}}-\Delta_{\mathrm{B}}^{\prime}-\Delta_{\mathrm{B}}
\end{array}\right)
\end{aligned}
$$

Let us first consider the $\delta$-peak contributions to the density of states. As seen previously (cf. (20)), they correspond to the eigenvalues of $g_{\mathrm{S}}$ and $g_{\mathrm{A}}$, that is

$$
\left.\begin{array}{l}
\mathscr{E}_{\mathrm{S}}=\frac{1}{2}\left(E_{\mathrm{A}}+E_{\mathrm{B}}+\Delta_{\mathrm{A}}^{\prime}+\Delta_{\mathrm{B}}^{\prime}-\Delta_{\mathrm{A}}-\Delta_{\mathrm{B}}\right) \pm\left[\frac{\left(E_{\mathrm{A}}-E_{\mathrm{B}}+\Delta_{\mathrm{A}}^{\prime}-\Delta_{\mathrm{B}}^{\prime}-\Delta_{\mathrm{A}}+\Delta_{\mathrm{B}}\right)^{2}}{4}+\Delta_{\mathrm{AB}}^{2}\right]^{1 / 2} \\
\mathscr{E}_{\mathrm{A}}=\frac{1}{2}\left(E_{\mathrm{A}}+E_{\mathrm{B}}-\Delta_{\mathrm{A}}^{\prime}-\Delta_{\mathrm{B}}^{\prime}-\Delta_{\mathrm{A}}-\Delta_{\mathrm{B}}\right) \pm\left[\frac{\left(E_{\mathrm{A}}-E_{\mathrm{B}}-\Delta_{\mathrm{A}}^{\prime}+\Delta_{\mathrm{B}}^{\prime}-\Delta_{\mathrm{A}}+\Delta_{\mathrm{B}}\right)^{2}}{4}+\Delta_{\mathrm{AB}}^{2}\right]^{1 / 2}
\end{array}\right\} .
$$

As for the energy bands, they are obtained by diagonalizing a series of subhamiltonians (25) associated with the eigenvalues $\varepsilon_{p}$ of the adjacency matrix, which here simplify into :

$$
h_{p}=\left(\begin{array}{l|l|l|l}
E_{\mathrm{A}}+\Delta_{\mathrm{A}}^{\prime}+\Delta_{\mathrm{A}}+\frac{1}{2} \Delta_{\mathrm{A}} \varepsilon_{p} & \Delta_{\mathrm{AB}}\left(1+\frac{\varepsilon_{p}}{2}\right) & 2 \Delta_{\mathrm{A}}\left(1-\frac{\varepsilon_{p}^{2}}{16}\right)^{1 / 2} & 2 \Delta_{\mathrm{AB}}\left(1-\frac{\varepsilon_{p}^{2}}{16}\right)^{1 / 2} \\
\hline \Delta_{\mathrm{AB}}\left(1+\frac{\varepsilon_{p}}{2}\right) & E_{\mathrm{B}}+\Delta_{\mathrm{B}}^{\prime}+\Delta_{\mathrm{B}}+\frac{1}{2} \Delta_{\mathrm{B}} \varepsilon_{p} & 2 \Delta_{\mathrm{AB}}\left(1-\frac{\varepsilon_{p}^{2}}{16}\right)^{1 / 2} & 2 \Delta_{\mathrm{B}}\left(1-\frac{\varepsilon_{p}^{2}}{16}\right)^{1 / 2} \\
\hline 2 \Delta_{\mathrm{A}}\left(1-\frac{\varepsilon_{p}^{2}}{16}\right)^{1 / 2} & 2 \Delta_{\mathrm{AB}}\left(1-\frac{\varepsilon_{p}^{2}}{16}\right)^{1 / 2} & E_{\mathrm{A}}-\Delta_{\mathrm{A}}^{\prime}+\Delta_{\mathrm{A}}-\frac{1}{2} \Delta_{\mathrm{A}} \varepsilon_{p} & \Delta_{\mathrm{AB}}\left(1-\frac{\varepsilon_{p}}{2}\right) \\
\hline 2 \Delta_{\mathrm{AB}}\left(1-\frac{\varepsilon_{p}^{2}}{16}\right)^{1 / 2} & 2 \Delta_{\mathrm{B}}\left(1-\frac{\varepsilon_{p}^{2}}{16}\right)^{1 / 2} & \Delta_{\mathrm{AB}}\left(1-\frac{\varepsilon_{p}}{2}\right) & E_{\mathrm{B}}-\Delta_{\mathrm{B}}^{\prime}+\Delta_{\mathrm{B}}-\frac{1}{2} \Delta_{\mathrm{B}} \varepsilon_{p}
\end{array} \mid .\right.
$$

The new matrix elements in (67) are those which involve the $\Delta_{\mathrm{AB}}$ coupling constant between bonding and antibonding orbitals. Numerical computation, for $-4 \leqslant \varepsilon_{p} \leqslant 4$, yields the spectrum shown on figure 2 , where it is compared with the results of [10]. The band shifts obtained by taking the bondingantibonding interaction into account, while not modifying the general aspect of the spectrum, may reach $0.9 \mathrm{eV}$. What is more important is that other interactions neglected by Lannoo and Bensoussan, and particularly those which involve the $\tau$ orbitals (which are essential here since they give rise to the valence band), could be brought into the picture without suppressing the factorization of the LCAO Hamiltonian.

Of course, numerical calculations are more complicated when more interactions are taken into account : in the same way, for crystals, the subhamiltonians associated with different wave-vectors are larger when more orbitals and more interactions are considered. But it is clear that our formalism provides a convenient tool for the treatment of complex practical problems.
6. Conclusion. - We have shown that the main result of the L.W.T. theorem may be extended to more general and complicated structures : in a wide range of cases, there is a possible factorization of the tight-binding Hamiltonian into a series of subhamiltonians, some of which yield delta peaks in the energy density of states, while each of the others is associated with one eigenvalue of the adjacency matrix of the graph underlying the whole structure, and may be calculated as a function of this eigenvalue.

As indicated before, the presentation we have here followed rested on the assumption that only nearest-neighbour interactions were involved. However it is an easy matter to get rid of this restriction.

Let us consider the first case corresponding to only one kind of vertex. The essential point in the foregoing demonstration was the fact that all three matrices $C, \Gamma_{\mathrm{S}} \Gamma_{\mathrm{S}}^{+}, \Gamma_{\mathrm{A}} \Gamma_{\mathrm{A}}^{+}$commute and can thus be diagonalized through the same unitary transformation. Introducing interactions between orbitals belonging to edges and vertices at larger distances on the graph G, provided these interactions still possess the essential 
property that they only depend on the type of orbitals considered and on their relative positions on the graph, results in the addition, to the various submatrices in the Hamiltonian (9), of new terms derived from the former ones by replacing the matrices $C$, $\Gamma_{\mathrm{s}}$ and $\Gamma_{\mathrm{A}}$ by their products by $C$ or various powers of this matrix. This modification is still consistent with the commutation conditions recalled above, so that the foregoing demonstration may be applied with only minor changes. The final subhamiltonians $h_{p}$ are, of course, somewhat more complicated since more interactions are taken into account, but the essential result remains, namely the factorization of the Hamiltonian.

An analogous generalization obtains when vertices belong to two different types.

Finally, we should stress that, while, in order to be more specific, we have treated here the problem of a tight-binding electronic Hamiltonian, immediate transposition is possible to other physical situations involving reticular vibrations [13], magnetic excitations, etc...

\section{References}

[1] Weaire, D., Phys. Rev. Lett. 26 (1971) 1541.

[2] Thorpe, M. F. and Weaire, D., Phys. Rev. B 4 (1971) 2508 ; B 4 (1971) 3518.

[3] Leman, G., Ann. Phys. 7 (1962) 505.

[4] HaRARY, F., Graph Theory and Theoretical Physics (Academic Press) 1967.

[5] Weaire, D. and Thorpe, M. F., Bull. Am. Phys. Soc. 17 (1972) 274 and Electronic Structure of Amorphous Semiconductors, in Computational Methods for Large Molecules and Localized States in Solids, eds. Herman, McLean, Nesbet (Plenum Press) 1973.

[6] Straley, J. P., Phys. Rev. B 6 (1972) 4086.
[7] Hulin, M., Phys. Status Solidi (b) 52 (1972) 119.

[8] Streitwolf, H. W., Phys. Status Solidi (b) 63 (1974) 529.

[9] Lannoo, M. and Bensoussan, M., Proceedings of the 7th International Conference on Amorphous and Liquid Semiconductors (Edinburgh 1977) p. 291.

[10] Lannoo, M. and Bensoussan, M., Phys. Rev. B 16 (1977) 3546.

[11] Halmos, P., Finite-dimensional Vector Spaces (Van Nostrand) $1958, \S 53$.

[12] Chen, W. K., Applied Graph Theory (North-Holland) 1971, Chap. 2.

[13] Sen, P. N. and ThOrPe, M. F., Phys. Rev. B 15 (1977) 4030. 